\title{
THE FUNDAMENTAL LEMMA OF COMPLEXITY FOR ARBITRARY FINITE SEMIGROUPS ${ }^{1}$
}

\author{
BY JOHN RHODES ${ }^{2}$
}

Communicated by Saunders MacLane, June 26, 1968

1. Statement of the results and some corollaries. All semigroups considered are of finite order. In the recent paper [3] and in the recent book [2] the complexity of a semigroup was defined and definitive results were obtained for determining the complexity of a semigroup which was the union of groups. Herein we state generalizations, valid for arbitrary finite semigroups, of those previous results. All undefined notation is explained in [2].

We first recall the definition of complexity. See also [2] or [3]. One semigroup, $S_{1}$, is said to divide another semigroup, $S_{2}$, if and only if $S_{1}$ is a homomorphic image of a subsemigroup $S \leqq S_{2}$. If $S$ is a semigroup, $\operatorname{Endo}(S)$ denotes the semigroup of endomorphisms of $S$ under composition. If $S_{1}$ and $S_{2}$ are semigroups and $Y$ is a homomorphism of $S_{1}$ into Endo $\left(S_{2}\right)$, the semidirect product of $S_{2}$ by $S_{1}$ with connecting homomorphism $Y$, denoted by $S_{2} \times_{Y} S_{1}$, is the semigroup with elements $S_{2} \times S_{1}$ and product defined by $\left(s_{2}, s_{1}\right) \cdot\left(s_{2}^{\prime}, s_{1}^{\prime}\right)=\left(s_{2} \cdot Y\left(s_{1}\right)\left(s_{2}^{\prime}\right)\right.$, $\left.s_{1} \cdot s_{1}^{\prime}\right)$.

We can construct new semigroups from old ones by taking semidirect products and then divisors. $S_{n} \times_{Y_{n-1}} \cdots \times_{Y_{2}} S_{2} \times_{Y_{1}} S_{1}$ denotes $\left.\left(\cdots\left(S_{n} \times_{Y_{n-1}} S_{n-1}\right) \times_{Y_{n-2}} S_{n-2}\right) \cdots \times_{Y_{1}} S_{1}\right)$ where $Y_{n-2}$ is a homomorphism of $S_{n-2}$ into $\operatorname{Endo}\left(S_{n} \times_{Y_{n-1}} S_{n-1}\right)$, etc. We say $S$ is a combinatorial semigroup if and only if the subsemigroups of $S$ which are groups are singletons. The main theorem of [1] (see also [2, Chapter 5]) implies that for each semigroup $S$ there exist semigroups $S_{n}, \cdots, S_{1}$ and connecting homomorphisms $Y_{n-1}, \cdots, Y_{1}$ so that

$$
S \text { divides } S_{n} \times_{Y_{n-1}} \cdots \times_{Y_{1}} S_{1}
$$

and $S_{k}$ is either a simple nontrivial group dividing $S$ or $S_{k}$ is a combinatorial semigroup, for $k=1, \cdots, n$.

$\#_{G}(S)$, the (group) complexity of $S$, is by definition the smallest nonnegative integer $n$ such that

1 This research was sponsored in part by the United States Air Force, Office of Scientific Research, Grant Number AF-AFOSR-68-1477.

${ }^{2}$ Alfred P. Sloan Research Fellow. 


$$
\begin{aligned}
S \text { divides } C_{n} \times_{Y_{n-1}} G_{n} \times_{z_{n-1} C_{n-1}} \times_{Y_{n-2} G_{n-1}} \times_{z_{n-2}} \cdots \\
C_{1} \times_{Y_{0}} G_{1} \times_{z_{0}} C_{0}
\end{aligned}
$$

with $C_{n}, \cdots, C_{1}, C_{0}$ combinatorial semigroups and $G_{n}, \cdots, G_{1}$ nontrivial groups. For extensive background see [2].

Let $S_{F}$ denote the collection of all finite semigroups, $S$ the collection of all finite semigroups which are union of groups and $N$ the nonnegative integers. Then $\#_{G}: S_{F} \rightarrow N$. In [3] and [2, Chapter 9], it was proved that $\#_{G}$ restricted to $S$ satisfies the following axioms:

Axiom I. \#G $(S)=\max \left\{\#_{G}\left(S_{i}\right): i=i, \cdots, n\right\}$ if $S \leqq \leqq S_{1} \times \cdots \times S_{n}$ where $\leqq \leqq$ denotes subdirect product. See [2].

Axiom II. (FundAMENTAL LEMMA OF COMPLEXITy). Let $I$ be a combinatorial ideal of $S$. Then

$$
\#_{G}(S)=\#_{G}(S / I) \text {. Also } \#_{G}(\{0\})=0 \text {. }
$$

Axiom III. Let $S \neq\{0\}$ and let $S$ be a group mapping (GM) semigroup with RLM the right letter mapping homomorphic image of $S .{ }^{3}$ Then

$$
\#_{G}(S)=\#_{G}(\operatorname{RLM}(S))+1 .
$$

We ask which Axioms remain valid for $\#_{G}: \varsigma_{F} \rightarrow N$ ?

It is trivial to verify that Axiom I remains valid for $S_{F}$. It is easy to see that Axiom III is false for $\mathcal{S}_{F}$, e.g. the symmetric inverse semigroup on $n$ letters has complexity 1 . See [7]. In fact, no function from $S_{F}$ into $N$ satisfies all three Axioms. In [2, Corollary 9.3.4], Axiom II is proved to be equivalent to Axiom $\mathrm{II}^{\prime}$.

AxIom II'. Let the epimorphism $\theta: S \rightarrow T$ be one-to-one when restricted to each subgroup of $S$. Then $\#_{G}(S)=\#_{G}(T)$.

The epimorphisms of the hypothesis of Axiom II' are called $\gamma$-epimorphisms in [2]. Our main result is the following theorem.

Theorem. Axiom II, or equivalently, Axiom II' holds for all finite semigroups.

It is well known (see [2, Proposition 8.2.17(b)]) that if $S$ is a GM semigroup then either $\#_{a}(S)$ equals $\#_{G}(\operatorname{RLM}(S))+1$ or $\#_{G}(\operatorname{RLM}(S))$. We say $S$ is a pure group mapping (PGM) semigroup if and only if $S$ is a GM semigroup $\neq\{0\}$ and (1.4) holds for $S$.

${ }^{3} S$ is a GM semigroup iff $S$ has a 0 -minimal noncombinatorial ideal $I$ so that $S$ acts faithfully on $I$ by right multiplication and also by left multiplication. $\operatorname{RLM}(S)$ is the action made faithful of $S$ by right multiplication on the principal left ideals of $I$. See [2]. 
CoRollary 1. \# $G(S)$ equals the largest nonnegative integer $n=\#_{1}(S)$ such that there exists a series

$$
\begin{aligned}
S \rightarrow \rightarrow \mathrm{PGM}_{1} \rightarrow \rightarrow \operatorname{RLM}\left(\mathrm{PGM}_{1}\right) & \rightarrow \cdots \\
& \rightarrow \longrightarrow \mathrm{PGM}_{n} \rightarrow \rightarrow \operatorname{RLM}\left(\mathrm{PGM}_{n}\right)
\end{aligned}
$$

where $\rightarrow \rightarrow$ denotes epimorphism, and $\mathrm{PGM}_{k}$ denotes a $\mathrm{PGM}$ semigroup $\neq\{0\}$ for $k=1, \cdots, n$.

Proof. First \# $\#_{1}(S) \leqq \# \theta(S)$ follows by the definition of PGM. The reverse inequality $\#_{G}(S) \leqq \#_{1}(S)$ follows from [2, Lemma 8.2.19(b)], Axiom II and the definition of PGM. See the proof of $[2$, Theorem 9.2.5].

\section{CoRollary 2.}

$$
S_{\mathfrak{L}} \rightarrow T^{4} \text { implies } \# G(T) \leqq \# G(S) \leqq \# G(T)+1 .
$$

Proof. $S \rightarrow \rightarrow T_{\mathscr{L}^{\circ}}$ the minimal $\mathfrak{L}^{\prime}$ homomorphic image of $S$ equals $S \rightarrow \rightarrow S^{\mathrm{RLM}}$ by [2, Fact 8.3.9(c)]. Now apply Corollary 1 .

CoRollary 3. (Continuity OF COMPLEXITy With RESPECT TO HOMOMORPHISMS) Let $\theta: S \rightarrow \rightarrow T$ be an epimorphism, and let \# $\#_{a}(S)=n$ and \# $\#_{G}(T)=k$. Then there exists epimorphisms $S=S_{n} \rightarrow \rightarrow S_{n-1}$ $\rightarrow \rightarrow \cdots \rightarrow \rightarrow S_{k}=T$, so that the composite epimorphism is $\theta$, and $\# G\left(S_{j}\right)=j$ for $j=k, \cdots, n$.

Proof. Apply [2, Theorem 8.1.14], the Theorem and Corollary 2.

Corollary 4. \#G $(S)$ equals the maximum of the $\#_{G}\left(S^{\prime}\right)$ where $S^{\prime}$ ranges over the $\phi(S)$ where $\phi$ is an irreducible representation of $S$ into $n \times n$ complex matrices.

Proof. The direct sum of the $\phi$ 's give a $\gamma$-epimorphism by [6].

2. Indication of the proof. Complete details will appear in [4]. Unfortunately they are long and messy. However, we will try to make the philosophy of the proof clear by the following discussion.

Suppose for each semigroup $S$ we can construct another semigroup $\alpha(S)$ such that

$$
\alpha(S) \rightarrow \rightarrow S
$$

and if $I$ is a combinatorial ideal of $S$ then

${ }^{4} \theta: S \rightarrow \rightarrow T$ is an $\mathscr{L}$ (resp. $\mathscr{L}^{\prime}$ ) epimorphism iff $s_{1}, s_{2} \in S$ (and $s_{1}, s_{2}$ regular elements) and $\theta_{1}\left(s_{1}\right)=\theta_{1}\left(s_{2}\right)$ implies $S^{1} s_{1}=S^{1} s_{2}$. See [2]. 


$$
\begin{aligned}
& \alpha(S) \text { divides } C \text { w } \alpha[(S / I)] \text { or at least } \\
& C(\alpha(S)) \leqq(C, 1) \oplus C(\alpha(S / I)) .{ }^{5}
\end{aligned}
$$

Then clearly to prove the Theorem it suffices to prove

$$
C(\alpha(S)) \leqq(C, 1) \oplus C(S)
$$

or equivalently by (2.1)

$$
C(\alpha(S)) \approx C(S)
$$

where $C(S) \approx C(T)$ iff $C(S) \leqq(\mathrm{C}, 1) \oplus C(T)$ and $C(T) \leqq(\mathrm{C}, 1) \oplus C(S)$.

EXAMPLES OF $\alpha$. Before continuing we list some good examples of $\alpha$. With reference to $[2, \S 5.4]$, we suppose that for each $S$ we choose a system of subsemigroups $S_{n}, \cdots, S_{1}$ and we let $\alpha(S)$ be the subsemigroup of

$$
\left(S_{n}^{I},\left(S_{n}^{I}\right) \sim\right) \mathrm{w} \cdots \mathrm{w}\left(S_{1}^{I},\left(S_{1}^{I}\right) \sim\right)
$$

generated by $\{\hat{s}: s \in S\}$ defined in the proof of Lemma 5.4.4 of [2]. Clearly (2.1) holds.

(2.3) If $S$ is a union of groups and the system is chosen to be the g-classes of $S$ as in Remark 5.4.14 of [2], then $\alpha(S)$ satisfies (2.2), as can be verified. See [3] or Chapter 9 of [2].

(2.4) If $I$ is a combinatorial ideal of $S$, then the system $S_{n}, \cdots, S_{1}$ can be chosen so that either $S_{i} \cap I=\varnothing$ or $S_{i}$ is combinatorial and contains $I$. In this case (2.2) can be verified. See [4] for complete details.

Yet another way to construct $\alpha$ 's is the following.

(2.5) Consider the right regular representation $\left(S^{I}, S\right)$ and apply the method of Zeiger (see [9] and Chapter 4 of [2]). Let $\alpha(S)$ be the subsemigroup of the wreath product of permutation-reset mapping semigroups so obtained which maps homomorphically onto $S$. Thus (2.1) holds and (2.2) can be verified. See [4] for complete details.

Now we give a method by which $\left(^{*}\right)$ can be proved. We first note that if $S$ is a union of groups and $\alpha$ is given by (2.3), then $(*)$ can be verified by brute force using the machine method of [1]. For the details see [3] or Chapter 9 of [2]. The general case seems difficult by direct methods and we proceed indirectly as follows.

- $S_{2}$ w $S_{1}$ denotes the wreath product of the right regular representation of $S_{1}$ by $S_{2}$, i.e., $\left.S_{2} \mathrm{w} S_{1}=\left(S_{2}{ }^{I}, S_{2}\right)\right\rangle\left(S_{1}{ }^{I}, S_{1}\right)$. Let $n=\#(S)$ be as defined just before (2.10). Then by definition $C(S)=(C, n)$, resp. $(G, n)$, resp. $(C \vee G, n)$ if $S$ satisfies (2.10) (b) and not (2.10) (a), resp. $S$ satisfies (2.10) (a) and not (2.10(b), resp. $S$ satisfies both (2.10) (a) and (b). By definition, $(C, 1) \oplus(C, n)=(C, 1) \oplus(C \vee G, n)=(C, 1) \oplus(G$, $n-1)=(C, n)$. Finally, by definition $(\alpha, v) \leqq(\beta, j)$ iff $v \leqq j$, or $v=j$ and $\alpha=\beta$, or $v=j$ and $\alpha=C \vee G$. See [2]. 
Suppose one can show

(2.6), (2.6)' (ENLARging Lemma). If $C(S) \approx C(T)$ and $S$ divides $T$ (resp. $T \rightarrow \rightarrow S$ ) and $\left({ }^{*}\right)$ holds for $T$, then $(*)$ holds for $S$.

(2.7) Let $\theta: S \longleftrightarrow \rightarrow T$ be a $\gamma(\mathcal{F C})$-epimorphism. ${ }^{6}$ Then $\alpha(S)$ divides $C$ w $\alpha(T)$ with $C$ combinatorial or at least

$$
C(\alpha(S)) \leqq(C, 1) \oplus C(\alpha(T)) .
$$

(2.8), (2.8)' Suppose $\theta: S \rightarrow \rightarrow T$ and $\theta$ is an $\mathscr{L}$ (resp. $\mathscr{L}^{\prime}$ ) epimorphism. Then $\alpha(S)$ divides $C_{1} \mathrm{w} G \mathrm{w} C_{2} \mathrm{w} \alpha(T)$ or at least $C(\alpha(S)) \leqq(C, 3)$ $\oplus C(\alpha(T))$.

Then

Lemma (2.9). (2.1), (2.6)-(2.8) or (2.1), (2.6)', (2.7) and $(2.8)^{\prime}$ imply $(*)$.

Proof. Suppose (2.9) is false and let $S$ be a counter-example whose complexity number (defined next) \#(S)=n is as small as possible. By the definition of complexity number \#(S) either

(2.10)(a) $S$ divides $G_{n}$ w $C_{n-1}$ w $G_{n-2}$ w $C_{n-2}$ w $\cdots=W$

or

(2.10)(b) $S$ divides $C_{n}$ w $G_{n-1}$ w $C_{n-2}$ w $G_{n-2} \mathrm{w} \cdots=W$

where $G_{j}$ 's are groups and the $C_{u}$ 's are combinatorial monoids and for no smaller $n$ is $(2.10)$ (a) or (b) true. But $C(S) \approx C(W)$ so (2.6) implies $\left(^{*}\right)$ is false for $W$. But in Case (2.10) (b)

$$
W \underset{\gamma(\mathfrak{F C})}{\longrightarrow} W_{-1}=p_{-1}(W)
$$

where $p_{-1}$ is the projection onto the first $n-1$ coordinates. In case (2.10) (a)

$$
W \underset{\mathfrak{L}}{\rightarrow} W_{-1}=p_{-1}(W)
$$

and in either case $\#\left(W_{-1}\right)=\#(W)-1=\#(S)-1$. Thus by induction ${ }^{*}$ ) holds for $W_{-1}$ and we have
(2.12)(a)
$C\left(W_{-1}\right)=(C, n-1)$
(2.12)(b)$$
C\left(W_{-1}\right)=(G, n-1)
$$

respectively. But then (2.11), (2.12) and (2.7) and (2.8) implies $\left({ }^{*}\right)$ holds for $W$, a contradiction. The other case with $(2.6)^{\prime}$, etc. proceeds similarly. This proves (2.9).

${ }^{\circ} \theta$ restricted to each $\mathcal{H}$-class of $S$ is one-to-one. 
Sketch OF THE PROOF OF THE THEOREM. Using $\alpha$ of (2.4) which we denote by $\alpha^{*}$ we verify (2.1) and (2.2) and further show that

$$
\alpha^{*}(S) \underset{\gamma(\mathfrak{H C})}{\longrightarrow} S
$$

We do not verify (2.6)-(2.8) directly for $\alpha^{*}$ of (2.4).

Then using $\alpha$ of (2.5) which we denote by $Z$, we verify (2.1) but not (2.2) for $Z$ because I can contain large nonregular H-classes of $S$. However, we can verify (2.6), (2.7) and (2.8) for $Z$ by using the classification of maximal proper epimorphisms proved in [5]. Then Lemma (2.9) implies (*) for $Z(S)$. Then (*) for $Z$ and (2.7) implies

$$
C(S) \approx C(T) \text { if } S \underset{\gamma(\mathcal{H C})}{\longrightarrow} T .
$$

But from the first paragraph

$$
\alpha^{*}(S) \underset{\gamma(\mathfrak{H C})}{\longrightarrow} S
$$

so $\left(^{*}\right)$ holds for $\alpha^{*}$, so (2.1), (2.2) and (*) holds for $\alpha^{*}$ and the Theorem follows.

For further results on complexity see [7].

\section{REFERENCES}

1. Kenneth Krohn and John Rhodes, Algebraic theory of machines. I. Prime decomposition theorem for finite semigroups and machines, Trans. Amer. Math. Soc. 116 (1965), 450-464.

2. Kenneth Krohn, John Rhodes and Bret Tilson, "Lectures on the algebraic theory of finite semigroups and finite state machines," Chapters 1, 5-9 (Chapter 6 with M. A. Arbib, in Algebraic theory of machines, languages, and semigroups, edited by M. A. Arbib, Academic Press, New York, 1968.

3. Kenneth Krohn and John Rhodes, Complexity of finite semigroups, Ann. of Math. 88 (1968), 128-160.

4. John Rhodes, $A$ proof of the fundamental lemma of complexity for arbitrary finite semigroups, to be submitted to Math. Systems Theory.

5. — A homomorphism theorem for finite semigroups, J. Math Systems Theory 1(1967), 289-304.

6. - Complexity and characters of finite semigroups. J. Combinatorial Theory (to appear).

7. John Rhodes and Bret Tilson, Lower bounds for complexity of finite semigroups, submitted to Math. Systems Theory.

8. H. P. Zeiger, Cascade synthesis of finite-state machines, Information and Control 10 (1967), 419-433, plus erratum.

University of California, Berkeley 